\title{
Lebesgue points for Sobolev functions on metric spaces
}

\section{Juha Kinnunen and Visa Latvala}

\begin{abstract}
Our main objective is to study the pointwise behaviour of Sobolev functions on a metric measure space. We prove that a Sobolev function has Lebesgue points outside a set of capacity zero if the measure is doubling. This result seems to be new even for the weighted Sobolev spaces on Euclidean spaces. The crucial ingredient of our argument is a maximal function related to discrete convolution approximations. In particular, we do not use the Besicovitch covering theorem, extension theorems or representation formulas for Sobolev functions.
\end{abstract}

\section{Introduction}

By the classical Lebesgue differentiation theorem almost every point is a Lebesgue point for a locally integrable function. It is natural to expect that if the function is more regular, the exceptional set is smaller. The main objective of our note is to study Lebesgue points for Sobolev functions on a metric measure space. The concept of capacity plays a key role in understanding the pointwise behaviour of Sobolev functions and it is a substitute for the measure in Lusin and Egorov type theorems. Sobolev functions are defined only up to a set of measure zero, but they can be defined pointwise up to a set of capacity zero. Indeed, every Sobolev function has a unique quasicontinuous representative for which there is a set of arbitrarily small capacity so that the function is continuous when restricted to the complement of the exceptional set. Our main theorem shows that Sobolev functions on a doubling metric measure space have Lebesgue points outside a set of

2000 Mathematics Subject Classification: 46E35.

Keywords: Sobolev spaces, spaces of homogeneous type, doubling measures, capacity, regularity, maximal functions. 
capacity zero and the quasicontinuous representative can be obtained by taking the limit of integral averages over small balls.

Recently there has been some interest in defining the first order Sobolev spaces in a very general context, see [3], [6], [8], [12], [13] and [21]. Our argument is based on a general principle and with suitable modifications it applies to any of the definitions. Our result seems to be new even for weighted Sobolev spaces on Euclidean spaces defined in [6] and [12]. The result has applications to the regularity theory for minimizers of variational integrals on metric measure spaces, see [2].

Standard proofs of refinements of Lebesgue's theorem are based on a capacitary weak type estimate for the Hardy-Littlewood maximal function, see [5], [7], [15], [19] or [22]. This estimate is usually proved by using the Besicovitch covering theorem, extension results or representation formulas for Sobolev functions. We do not have these tools available. In the classical case we can also use the fact that the Hardy-Littlewood maximal operator is bounded in the Sobolev space, see [16]. However, examples in [1] show that the Hardy-Littlewood maximal operator does not have the required regularity properties in metric spaces. Our proof is based on a construction of a maximal function which is related to discrete convolution approximations of the original function. The defined discrete maximal operator is smoother than the standard Hardy-Littlewood maximal operator and it can be used as a test function for the capacity.

For simplicity, we have chosen the definition of Sobolev spaces on a metric measure space due to Hajłasz [8]. A general outline of the theory and further references can be found in [11]. However, it is easy to modify our argument to cover the spaces defined in [3], [6], [12], [13] and [21]. Then we have to assume, in addition, that the space supports a Poincaré inequality. We leave the details for the interested reader.

Acknowledgements. The authors wish to thank Professor Juha Heinonen for fruitful discussions. A part of the research was done when the authors visited the Mittag-Leffler Institute. The authors wish to thank the Academy of Finland and the Institute for the support.

\section{Sobolev spaces on metric spaces}

In this section we recall the definition due to Hajłasz [8] of the first order Sobolev space on an arbitrary metric measure space. Let $(X, d)$ be a metric space and let $\mu$ be a non-negative Borel regular outer measure on $X$. In the following, we keep the metric measure space $(X, d, \mu)$ fixed, and for short, we denote it by $X$. The Lebesgue space $L^{p}(X)$ with $1<p<\infty$ is the Banach 
space of all $\mu$-a.e. defined $\mu$-measurable functions $u: X \rightarrow[-\infty, \infty]$ with the norm

$$
\|u\|_{L^{p}(X)}=\left(\int_{X}|u|^{p} d \mu\right)^{1 / p} .
$$

Let $1<p \leq \infty$ and suppose that $u \in L^{p}(X)$. We denote by $D(u)$ the set of all $\mu$-measurable functions $g_{u}: X \rightarrow[0, \infty]$ such that

$$
|u(x)-u(y)| \leq d(x, y)\left(g_{u}(x)+g_{u}(y)\right)
$$

for every $x, y \in X \backslash N, x \neq y$, with $\mu(N)=0$. In the metric setting, instead of having the gradient, we have the whole set $D(u)$ of maximal gradients of $u$. A function $u \in L^{p}(X)$ belongs to the Sobolev space $M^{1, p}(X)$ if $D(u) \cap L^{p}(X) \neq \emptyset$. The Sobolev space $M^{1, p}(X)$ is equipped with the norm

$$
\|u\|_{M^{1, p}(X)}=\left(\|u\|_{L^{p}(X)}^{p}+\|u\|_{L^{1, p}(X)}^{p}\right)^{1 / p},
$$

where

$$
\|u\|_{L^{1, p}(X)}=\inf \left\{\|g\|_{L^{p}(X)}: g \in D(u) \cap L^{p}(X)\right\} .
$$

We recall some basic properties of the Sobolev space $M^{1, p}(X)$. If $X=\mathbb{R}^{n}$ with the Euclidean metric and the Lebesgue measure, then

$$
M^{1, p}\left(\mathbb{R}^{n}\right)=W^{1, p}\left(\mathbb{R}^{n}\right), \quad 1<p \leq \infty .
$$

Moreover, the norms are comparable (see [8]). Here $W^{1, p}\left(\mathbb{R}^{n}\right)$ is the first order Sobolev space of functions in $L^{p}\left(\mathbb{R}^{n}\right)$, whose first distributional derivatives belong to $L^{p}\left(\mathbb{R}^{n}\right)$ with the norm

$$
\|u\|_{W^{1, p}\left(\mathbb{R}^{n}\right)}=\left(\|u\|_{L^{p}\left(\mathbb{R}^{n}\right)}^{p}+\|D u\|_{L^{p}\left(\mathbb{R}^{n}\right)}^{p}\right)^{1 / p} .
$$

Indeed, if $u \in W^{1, p}\left(\mathbb{R}^{n}\right)$, then we have the pointwise inequality

$$
|u(x)-u(y)| \leq c|x-y|(M|D u|(x)+M|D u|(y))
$$

for Lebesgue almost every $x, y \in \mathbb{R}^{n}$. Here $M|D u|$ is the Hardy-Littlewood maximal function of $|D u|$. The Hardy-Littlewood maximal function theorem implies that the maximal operator is bounded in $L^{p}\left(\mathbb{R}^{n}\right)$ when $1<p \leq \infty$. This shows that $M|D u| \in D(u) \cap L^{p}\left(\mathbb{R}^{n}\right)$ and hence $W^{1, p}\left(\mathbb{R}^{n}\right) \subset M^{1, p}\left(\mathbb{R}^{n}\right)$. The reverse inclusion follows from the characterization of $W^{1, p}\left(\mathbb{R}^{n}\right)$ with the integrated difference quotients. Since the maximal operator is not bounded in $L^{1}\left(\mathbb{R}^{n}\right)$ we exclude the case $p=1$ in the definition. This also suggests that $g_{u} \in D(u)$ corresponds to the maximal function of the gradient of $u$ rather than the gradient itself. 
The Sobolev space $M^{1, p}(X)$ with the norm (2.2) is a Banach space, see Theorem 3 in [8]. By Theorem 5 in [8] Lipschitz continuous functions are dense in $M^{1, p}(X)$ and hence $M^{1, p}(X)$ can be characterized as the completion of $C(X) \cap M^{1, p}(X)$ with respect to the norm (2.2).

If $u \in M^{1, p}(X)$ and $g_{u} \in D(u)$, then the Poincaré inequality

$$
f_{B(x, r)}\left|u-u_{B(x, r)}\right| d \mu \leq c r f_{B(x, r)} g_{u} d \mu
$$

holds for every $x \in X$ and $r>0$. Here we use the standard notation

$$
u_{B(x, r)}=f_{B(x, r)} u d \mu=\frac{1}{\mu(B(x, r))} \int_{B(x, r)} u d \mu
$$

and $B(x, r)$ denotes the open ball with the center $x$ and the radius $r>0$. The Poincaré inequality is easily proved by integrating the pointwise inequality (2.1) twice over the ball.

It is clear that if $g_{u} \in D(u)$ and $g_{v} \in D(v)$, then $g_{u}+g_{v} \in D(u+v)$. Moreover, it follows directly from $(2.1)$ and the triangle inequality that if $u \in M^{1, p}(X)$, then $|u| \in M^{1, p}(X)$ and that $D(u) \subset D(|u|)$. The following lemma is a version of the Leibniz differentiation rule, see Lemma 5.20 in [9].

Lemma 2.5 Let $u \in M^{1, p}(X)$ and $\phi$ be a bounded Lipschitz function. Then $u \phi \in M^{1, p}(X)$. Moreover, if $L$ is the Lipschitz constant of $\phi$ and $E \subset X$ such that $\phi=0$ in $X \backslash E$, then

$$
\left(g_{u}\|\phi\|_{\infty}+L|u|\right) \chi_{E} \in D(u \phi) \cap L^{p}(X)
$$

for every $g_{u} \in D(u) \cap L^{p}(X)$.

Proof. A straightforward computation shows that

$$
|u(x) \phi(x)-u(y) \phi(y)| \leq d(x, y)\left(\left(g_{u}(x)+g_{u}(y)\right)|\phi(x)|+L|u(y)|\right)
$$

and

$$
|u(x) \phi(x)-u(y) \phi(y)| \leq d(x, y)\left(\left(g_{u}(x)+g_{u}(y)\right)|\phi(y)|+L|u(x)|\right) .
$$

The claim follows from these inequalities easily.

It is also clear that the space $M^{1, p}(X)$ is closed under taking maximum and minimum over finitely many functions. The following simple lemma is a useful tool in showing that the supremum of countably many Sobolev functions belongs to the Sobolev space under some conditions. 
Lemma 2.6 Suppose that $u_{i}, i=1,2, \ldots$, are $\mu$-measurable functions, let $g_{i} \in D\left(u_{i}\right), i=1,2, \ldots$, and denote $g=\sup _{i} g_{i}$ and $u=\sup _{i} u_{i}$. Then $g \in D(u)$ provided $u<\infty \mu$-almost everywhere.

Proof. Let $x, y \in X \backslash N$ with $u(y) \leq u(x)<\infty$. Here $N$ is the union of exceptional sets for the functions $u_{i}$ as in (2.1). Let $\varepsilon>0$ and choose $i$ such that $u(x)<u_{i}(x)+\varepsilon$. Since $u(y) \geq u_{i}(y)$, we obtain

$$
\begin{aligned}
|u(x)-u(y)| & =u(x)-u(y) \leq u_{i}(x)+\varepsilon-u_{i}(y) \\
& \leq d(x, y)\left(g_{i}(x)+g_{i}(y)\right)+\varepsilon \leq d(x, y)(g(x)+g(y))+\varepsilon .
\end{aligned}
$$

Letting $\varepsilon \rightarrow 0$ we obtain the result.

2.7 Sobolev embeddings. A metric measure space $X$ is said to be $d o u$ bling if there is a constant $c_{\mu} \geq 1$ so that

$$
\mu(B(z, 2 r)) \leq c_{\mu} \mu(B(z, r))
$$

for every open ball $B(z, r)$ in $X$. The constant $c_{\mu}$ in (2.8) is called the doubling constant of $\mu$. Note that an iteration of the doubling property implies, that if $B(y, R)$ is a ball in $X, z \in B(y, R)$ and $0<r \leq R<\infty$, then

$$
\frac{\mu(B(z, r))}{\mu(B(y, R))} \geq c\left(\frac{r}{R}\right)^{Q}
$$

for some $c=c\left(c_{\mu}\right)$ and $Q=\log c_{\mu} / \log 2$. The exponent $Q$ serves as a counterpart of dimension related to the measure and, for example, in $\mathbb{R}^{n}$ with the Lebesgue measure $Q$ is equal to the dimension $n$.

A result of [10] (see also Theorem 5.1 in [11]) shows that in a doubling measure space a Poincaré inequality implies a Sobolev-Poincaré inequality. More precisely, if $1<p<Q$ and $1 \leq \kappa<Q /(Q-p)$, there is $c=c\left(p, \kappa, c_{\mu}\right)>$ 0 such that

$$
\left(f_{B(z, r)}\left|u-u_{B(z, r)}\right|^{\kappa p} d \mu\right)^{1 /(\kappa p)} \leq c r\left(f_{B(z, 5 r)} g_{u}^{p} d \mu\right)^{1 / p}
$$

for every $g_{u} \in D(u) \cap L^{p}(X)$. If $p>Q$, then

$$
|u(x)-u(y)| \leq c r^{Q / p} d(x, y)^{1-Q / p}\left(f_{B(z, 5 r)} g_{u}^{p} d \mu\right)^{1 / p}
$$

for every $x, y \in B(z, r) \backslash N$ with $\mu(N)=0$ and $g_{u} \in D(u) \cap L^{p}(X)$. In particular, this implies that, after a redefinition on a set of measure zero, functions in $M^{1, p}(X)$ with $p>Q$ are Hölder continuous on bounded subsets of $X$. See also [9]. In the borderline case $p=Q$ there is an exponential estimate, but we do not need it here. These are the counterparts of Sobolev embedding theorems on a metric measure space. 


\section{Maximal operator on Sobolev spaces}

Let $r>0$. We are interested in approximating the function $u$ at the scale of $3 r$. We begin by constructing a family of balls which cover the space and which do not overlap too much. Indeed, there is a family of balls $B\left(x_{i}, r\right)$, $i=1,2, \ldots$, such that

$$
X \subset \bigcup_{i=1}^{\infty} B\left(x_{i}, r\right)
$$

and

$$
\sum_{i=1}^{\infty} \chi_{B\left(x_{i}, 6 r\right)} \leq c<\infty .
$$

This means that the dilated balls $B\left(x_{i}, 6 r\right)$ are of bounded overlap. The constant $c$ depends only on the doubling constant $c_{\mu}$ and, in particular, it is independent of $r$.

Then we construct a partition of unity subordinate to the cover $\left\{B\left(x_{i}, r\right)\right\}$ of $X$. There is a family of functions $\phi_{i}, i=1,2, \ldots$, on $X$ such that $0 \leq \phi_{i} \leq 1, \phi_{i}=0$ on $X \backslash B\left(x_{i}, 6 r\right), \phi_{i} \geq c$ on $B\left(x_{i}, 3 r\right), \phi_{i}$ is Lipschitz with constant $c / r_{i}$ with $c$ depending only on the doubling constant, and

$$
\sum_{i=1}^{\infty} \phi_{i}=1
$$

on $X$. The partition of unity can be constructed by first choosing auxiliary cutoff functions $\widetilde{\phi}_{i}$ so that $0 \leq \widetilde{\phi}_{i} \leq 1, \widetilde{\phi}_{i}=0$ on $X \backslash B\left(x_{i}, 6 r\right), \widetilde{\phi}_{i}=1$ on $B\left(x_{i}, 3 r\right)$ and each $\widetilde{\phi}_{i}$ is Lipschitz with constant $c / r$. We can for example take

$$
\widetilde{\phi}_{i}(x)= \begin{cases}1, & x \in B\left(x_{i}, 3 r\right), \\ 2-\frac{d\left(x, x_{i}\right)}{3 r}, & x \in B\left(x_{i}, 6 r\right) \backslash B\left(x_{i}, 3 r\right), \\ 0, & x \in X \backslash B\left(x_{i}, 6 r\right) .\end{cases}
$$

Then we can define the functions $\phi_{i}, i=1,2, \ldots$, in the partition of unity by

$$
\phi_{i}(x)=\frac{\widetilde{\phi}_{i}(x)}{\sum_{j=1}^{\infty} \widetilde{\phi}_{j}(x)} .
$$

It is not hard to see that the defined functions satisfy the required properties.

Now we are ready to define the approximation of $u$ at the scale of $3 r$ by setting

$$
u_{r}(x)=\sum_{i=1}^{\infty} \phi_{i}(x) u_{B\left(x_{i}, 3 r\right)}
$$


for every $x \in X$. Sometimes $u_{r}$ is called the discrete convolution of $u$. The partition of unity and the discrete convolution are standard tools in harmonic analysis on homogeneous spaces, see for example [4] and [18]. See also pages $290-292$ of [20].

Let $r_{j}, j=1,2, \ldots$, be an enumeration of the positive rationals. For every radius $r_{j}$ we choose a covering $\left\{B\left(x_{i}, r_{j}\right)\right\}, i=1,2, \ldots$, of $X$ as above. Observe that for each radius there are many possible choices for the covering but we simply take one of those. We define the discrete maximal function related to the coverings $\left\{B\left(x_{i}, r_{j}\right)\right\}, i, j=1,2, \ldots$, by

$$
M^{*} u(x)=\sup _{j}|u|_{r_{j}}(x)
$$

for every $x \in X$. We emphasize the fact that the defined maximal operator depends on the chosen coverings. This does not matter, since we prove and use estimates which are independent of the coverings.

As a supremum of continuous functions, the discrete maximal function is lower semicontinuous and hence measurable. We observe that the defined maximal function is equivalent to the Hardy-Littlewood maximal function

$$
M u(x)=\sup _{r>0} f_{B(x, r)}|u| d \mu,
$$

which is a commonly used tool in analysis.

Lemma 3.1 There is a constant $c \geq 1$, which depends only on the doubling constant, such that

$$
c^{-1} M u(x) \leq M^{*} u(x) \leq c M u(x)
$$

for every $x \in X$.

Proof. We begin by proving the second inequality. Let $x \in X$ and $r_{j}$ be a positive rational number. Since $\phi_{i}=0$ on $X \backslash B\left(x_{i}, 6 r_{j}\right)$ and $B\left(x_{i}, 3 r_{j}\right) \subset$ $B\left(x, 9 r_{j}\right)$ for every $x \in B\left(x_{i}, 6 r_{j}\right)$, we have by the doubling condition (2.8) that

$$
\begin{aligned}
|u|_{r_{j}}(x) & =\sum_{i=1}^{\infty} \phi_{i}(x)|u|_{B\left(x_{i}, 3 r_{j}\right)} \\
& \leq \sum_{i=1}^{\infty} \phi_{i}(x) \frac{\mu\left(B\left(x, 9 r_{j}\right)\right)}{\mu\left(B\left(x_{i}, 3 r_{j}\right)\right)} f_{B\left(x, 9 r_{j}\right)}|u| d \mu \leq c M u(x),
\end{aligned}
$$

where $c$ depends only on the doubling constant $c_{\mu}$. The second inequality follows by taking the supremum on the left side. 
To prove the first inequality we observe that for each $x \in X$ there exists $i=i_{x}$ such that $x \in B\left(x_{i}, r_{j}\right)$. This implies that $B\left(x, r_{j}\right) \subset B\left(x_{i}, 2 r_{j}\right)$ and hence

$$
\begin{aligned}
f_{B\left(x, r_{j}\right)}|u| d \mu & \leq c f_{B\left(x_{i}, 3 r_{j}\right)}|u| d \mu \\
& \leq c \phi_{i}(x) f_{B\left(x_{i}, 3 r_{j}\right)}|u| d \mu \leq c M^{*} u(x) .
\end{aligned}
$$

In the second inequality we used the fact that $\phi_{i} \geq c$ on $B\left(x_{i}, r_{j}\right)$. Again the claim follows by taking the supremum on the left side.

By the Hardy-Littlewood maximal function theorem for doubling measures (see [4]) we see that the Hardy-Littlewood maximal operator is bounded on $L^{p}(X)$ when $1<p \leq \infty$ and maps $L^{1}(X)$ into the weak $L^{1}(X)$. Since the maximal operators are comparable we conclude that the same results hold for the discrete maximal operator $M^{*}$. In particular, there is a constant $c=c\left(p, c_{\mu}\right)>0$ such that

$$
\left\|M^{*} u\right\|_{L^{p}(X)} \leq c\|M u\|_{L^{p}(X)} \leq c\|u\|_{L^{p}(X)}
$$

whenever $p>1$.

Our goal is to show that the operator $M^{*}$ preserves the smoothness of the function in the sense that it is a bounded operator in $M^{1, p}(X)$. We begin by proving the corresponding result in a frozen scale.

Lemma 3.3 Suppose that $u \in M^{1, p}(X)$ with $p>1$ and let $r>0$. Then $u_{r} \in M^{1, p}(X)$ and

$$
c M g_{u} \in D\left(u_{r}\right) \cap L^{p}(X)
$$

for every $g_{u} \in D(u) \cap L^{p}(X)$. Here $c$ depends only on the doubling constant $c_{\mu}$.

Proof. We have

$$
u_{r}(x)=\sum_{i=1}^{\infty} \phi_{i}(x) u_{B\left(x_{i}, 3 r\right)}=u(x)+\sum_{i=1}^{\infty} \phi_{i}(x)\left(u_{B\left(x_{i}, 3 r\right)}-u(x)\right) .
$$

Observe that at each $x$ the sum is only over finitely many balls so that the convergence of the series is clear. This implies that

$$
g_{u}+\sum_{i=1}^{\infty} g_{\phi_{i}\left(u_{B\left(x_{i}, 3 r\right)}-u\right)} \in D\left(u_{r}\right)
$$


where by Lemma 2.5 we have

$$
\left(\frac{c}{r}\left|u-u_{B\left(x_{i}, 3 r\right)}\right|+g_{u}\right) \chi_{B\left(x_{i}, 6 r\right)} \in D\left(\phi_{i}\left(u_{B\left(x_{i}, 3 r\right)}-u\right)\right) .
$$

Here we also used the fact that $0 \leq \phi_{i} \leq 1$ for every $i=1,2, \ldots$ From this we conclude that

$$
g_{u}+\sum_{i=1}^{\infty}\left(\frac{c}{r}\left|u-u_{B\left(x_{i}, 3 r\right)}\right|+g_{u}\right) \chi_{B\left(x_{i}, 6 r\right)} \in D\left(u_{r}\right) .
$$

Let $x \in B\left(x_{i}, 6 r\right)$. Then $B\left(x_{i}, 3 r\right) \subset B(x, 9 r)$ and

$$
\left|u(x)-u_{B\left(x_{i}, 3 r\right)}\right| \leq\left|u(x)-u_{B(x, 9 r)}\right|+\left|u_{B(x, 9 r)}-u_{B\left(x_{i}, 3 r\right)}\right| .
$$

We estimate the second term on the right side by the Poincaré inequality (2.4) and the doubling condition (2.8) as

$$
\begin{aligned}
\left|u_{B(x, 9 r)}-u_{B\left(x_{i}, 3 r\right)}\right| \leq f_{B\left(x_{i}, 3 r\right)}\left|u-u_{B(x, 9 r)}\right| d \mu \\
\quad \leq c f_{B(x, 9 r)}\left|u-u_{B(x, 9 r)}\right| d \mu \leq c r f_{B(x, 9 r)} g_{u} d \mu .
\end{aligned}
$$

The first term on the right side of (3.5) is estimated by a standard telescoping argument. Since $\mu$-almost every point is a Lebesgue point for $u$, we have

$$
\begin{aligned}
\mid u(x) & -u_{B(x, 9 r)}\left|\leq \sum_{j=0}^{\infty}\right| u_{B\left(x, 3^{2-j} r\right)}-u_{B\left(x, 3^{1-j} r\right)} \mid \\
& \leq \sum_{j=0}^{\infty} f_{B\left(x, 3^{1-j} r\right)}\left|u-u_{B\left(x, 3^{2-j} r\right)}\right| d \mu \\
& \leq c \sum_{j=0}^{\infty} f_{B\left(x, 3^{2-j} r\right)}\left|u-u_{B\left(x, 3^{2-j} r\right)}\right| d \mu \\
& \leq c \sum_{j=0}^{\infty} 3^{2-j} r f_{B\left(x, 3^{2-j} r\right)} g_{u} d \mu \leq c r M g_{u}(x)
\end{aligned}
$$

for $\mu$-almost every $x \in X$. Here we used the Poincaré inequality and the doubling condition again.

Hence by (3.5) and the definition of the Hardy-Littlewood maximal function we have

$$
\left|u(x)-u_{B\left(x_{i}, 3 r\right)}\right| \leq c r f_{B(x, 9 r)} g_{u} d \mu+\operatorname{crMg} g_{u}(x) \leq \operatorname{crMg} g_{u}(x)
$$

for $\mu$-almost every $x \in X$. 
We observe that $g_{u}(x) \leq M g_{u}(x)$ for $\mu$-almost every $x \in X$ by the Lebesgue density theorem for doubling measures and using (3.4) we see that $c M g_{u} \in D\left(u_{r}\right)$ with $c$ depending only on the doubling constant. The maximal function theorem shows that there is $c=c\left(p, c_{\mu}\right)>0$ such that

$$
\left\|M g_{u}\right\|_{L^{p}(X)} \leq c\left\|g_{u}\right\|_{L^{p}(X)}
$$

and hence $c M g_{u} \in D\left(u_{r}\right) \cap L^{p}(X)$ for $p>1$. By Lemma 3.1 we have $u_{r} \leq c M u$ from which we conclude that $u_{r} \in L^{p}(X)$ by the maximal function theorem. This completes the proof.

Now we are ready to conclude that the maximal operator $M^{*}$ preserves the Sobolev space.

Theorem 3.6 Suppose that $u \in M^{1, p}(X)$ with $p>1$. Then $M^{*} u \in M^{1, p}(X)$ and

$$
c M g_{u} \in D\left(M^{*} u\right) \cap L^{p}(X)
$$

where $g_{u} \in D(u) \cap L^{p}(X)$ and $c>0$ depends only on the doubling constant.

Proof. By (3.2) we see that $M^{*} u \in L^{p}(X)$ and, in particular, $M^{*} u<\infty$ $\mu$-almost everywhere. The claim follows directly from Lemma 2.6, since $c M g_{u} \in D\left(u_{r_{j}}\right) \subset D\left(|u|_{r_{j}}\right)$ for every $j=1,2, \ldots$

\section{Remarks 3.7}

(1) Since the maximal operators $M^{*}$ and $M$ are equivalent by Lemma 3.1, we see that we can replace the Hardy-Littlewood maximal operator in the claims of Lemma 3.3 and Theorem 3.6 by $M^{*}$.

(2) By Theorem 3.6 and the Hardy-Littlewood maximal theorem we conclude that the discrete maximal operator $M^{*}$ is bounded in $M^{1, p}(X)$.

(3) Classical maximal function arguments show that $u_{r} \rightarrow u$ pointwise $\mu$-almost everywhere as $r \rightarrow 0$. A similar argument as in the proof of Lemma 3.3 shows that $u_{r} \rightarrow u$ in $M^{1, p}(X)$ as $r \rightarrow 0$. Hence the discrete convolution approximates the function also in the Sobolev norm.

\section{Lebesgue theorem for Sobolev functions}

There is a natural capacity in the Sobolev space. For $1<p<\infty$, the Sobolev $p$-capacity of the set $E \subset X$ is the number

$$
\mathrm{C}_{p}(E)=\inf \left\{\|u\|_{M^{1, p}(X)}^{p}: u \in \mathcal{A}(E)\right\},
$$

where

$$
\mathcal{A}(E)=\left\{u \in M^{1, p}(X): u \geq 1 \text { on a neighbourhood of } E\right\} .
$$


If $\mathcal{A}(E)=\emptyset$, we set $\mathrm{C}_{p}(E)=\infty$. The Sobolev capacity is a monotone and countably subadditive set function, see [17]. It is easy to see (Remark 3.3 in [17]) that the Sobolev capacity is an outer capacity, which means that

$$
\mathrm{C}_{p}(E)=\inf \left\{\mathrm{C}_{p}(O) O \supset E, O \text { open }\right\}
$$

The capacity measures the exceptional sets for Sobolev functions. To tell what we mean by this we need a definition. A function $u X \rightarrow[-\infty, \infty]$ is $p$ quasicontinuous in $X$ if for every $\varepsilon>0$ there is a set $E$ such that $\mathrm{C}_{p}(E)<\varepsilon$ and the restriction of $u$ to $X \backslash E$ is continuous. By outer regularity, we may assume that $E$ is open. Functions in $M^{1, p}(X)$ are defined only up to a set of measure zero, but the following result (Corollary 3.7 in [17]) shows that we may talk about the values of Sobolev functions outside a set of capacity zero.

Theorem 4.1 For each $u \in M^{1, p}(X)$ there is a p-quasicontinuous function $v \in M^{1, p}(X)$ such that $u=v \mu$-a.e. in $X$.

Moreover, the quasicontinuous representative is unique in the sense that if two quasicontinuous functions coincide $\mu$-almost everywhere, then they actually coincide outside a set of capacity zero. For a very nice proof of this we refer to [14].

Our objective is to show that the quasicontinuous representative can be obtained explicitely by looking at the integral averages of the function over small balls. We begin by proving a measure theoretic lemma. Roughly speaking it says that the capacity of the set where an integrable function is large is small. The proof is an easy modification of the corresponding result for Hausdorff measures, see for example Theorem 3 on page 77 of [5]. Since we do not know the measure of the ball, we do not get the Hausdorff measure estimate. The key point is that we have an estimate for the capacity of a ball. By ([17], Theorem 4.6) there is a constant $c=c\left(p, c_{\mu}\right)$ such that

$$
\mathrm{C}_{p}(B(x, r)) \leq c r^{-p} \mu(B(x, r)), \quad 0<r \leq 1 .
$$

The proof of (4.2) is not difficult: We simply test the capacity by a Lipschitz cutoff function which vanishes outside the ball $B(x, 2 r)$.

Lemma 4.3 Let $1<p<\infty$, suppose that $g \in L^{p}(X)$ with $g \geq 0$, and define

$$
E=\left\{x \in X: \limsup _{r \rightarrow 0} r^{p} f_{B(x, r)} g^{p} d \mu>0\right\} .
$$

Then $\mathrm{C}_{p}(E)=0$. 
Proof. Let $\varepsilon>0$ and

$$
E_{\varepsilon}=\left\{x \in X: \limsup _{r \rightarrow 0} r^{p} f_{B(x, r)} g^{p} d \mu>\varepsilon\right\} .
$$

We show that $\mathrm{C}_{p}\left(E_{\varepsilon}\right)=0$ for every $\varepsilon>0$, then the claim follows by subadditivity. Let $0<\delta<1$. For every $x \in E_{\varepsilon}$ there is $r_{x}$ with $0<r_{x}<\delta$ such that

$$
r_{x}^{p} f_{B\left(x, r_{x}\right)} g^{p} d \mu>\varepsilon .
$$

By the Vitali covering theorem (see [4]), there exists a subfamily of countably many pairwise disjoint balls $B\left(x_{i}, r_{i}\right), i=1,2, \ldots$, such that

$$
E_{\varepsilon} \subset \bigcup_{i=1}^{\infty} B\left(x_{i}, 5 r_{i}\right)
$$

Hence by subadditivity of the capacity, estimate (4.2) and the doubling condition we have

$$
\begin{aligned}
\mathrm{C}_{p}\left(E_{\varepsilon}\right) & \leq \sum_{i=1}^{\infty} \mathrm{C}_{p}\left(B\left(x_{i}, 5 r_{i}\right)\right) \leq c \sum_{i=1}^{\infty}\left(5 r_{i}\right)^{-p} \mu\left(B\left(x_{i}, 5 r_{i}\right)\right) \\
& \leq c \sum_{i=1}^{\infty} r_{i}^{-p} \mu\left(B\left(x_{i}, r_{i}\right)\right) \leq \frac{c}{\varepsilon} \sum_{i=1}^{\infty} \int_{B\left(x_{i}, r_{i}\right)} g^{p} d \mu \\
& =\frac{c}{\varepsilon} \int_{\bigcup_{i=1}^{\infty} B\left(x_{i}, r_{i}\right)} g^{p} d \mu .
\end{aligned}
$$

Here $c=c\left(p, c_{\mu}\right)>0$. Finally we observe that by the disjointness of the balls

$$
\mu\left(\bigcup_{i=1}^{\infty} B\left(x, r_{i}\right)\right)=\sum_{i=1}^{\infty} \mu\left(B\left(x_{i}, r_{i}\right)\right) \leq \sum_{i=1}^{\infty} \frac{r_{i}^{p}}{\varepsilon} \int_{B\left(x, r_{i}\right)} g^{p} d \mu \leq \frac{\delta^{p}}{\varepsilon} \int_{X} g^{p} d \mu
$$

which tends to zero as $\delta \rightarrow 0$. Hence the claim follows by absolute continuity of the integral.

The following capacitary weak type estimate for the maximal function is a crucial tool in the proof of the Lebesgue point theorem for Sobolev functions.

Lemma 4.4 Suppose that $u \in M^{1, p}(X)$ with $p>1$. Then

$$
\mathrm{C}_{p}(\{x \in X M u(x)>\lambda\}) \leq c \lambda^{-p}\|u\|_{M^{1, p}(X)}^{p}
$$

for every $\lambda>0$ with $c=c\left(p, c_{\mu}\right)>0$. 
Proof. By Lemma 3.1 we have

$$
\{x \in X M u(x)>\lambda\} \subset E_{\lambda},
$$

where $E_{\lambda}=\left\{x \in X c M^{*} u(x)>\lambda\right\}$ is open by lower semicontinuity of $M^{*} u$ and $c$ is a constant depending only on the doubling constant such that $c M^{*} u \geq M u$. The function $c M^{*} u / \lambda$ is admissible for $E_{\lambda}$.

Therefore from Theorem 3.6 we conclude that

$$
\begin{gathered}
\mathrm{C}_{p}\left(E_{\lambda}\right) \leq\left\|c M^{*} u / \lambda\right\|_{M^{1, p}(X)}^{p} \leq c \lambda^{-p}\left(\left\|M^{*} u\right\|_{L^{p}(X)}^{p}+\left\|M g_{u}\right\|_{L^{p}(X)}^{p}\right) \\
\leq c \lambda^{-p}\left(\|u\|_{L^{p}(X)}^{p}+\left\|g_{u}\right\|_{L^{p}(X)}^{p}\right) .
\end{gathered}
$$

The claim follows by taking the infimum over all maximal gradients of $u$ on the right side.

Now we are ready to prove our main result. If $u \in M^{1, p}(X)$ with $p>Q$, then using (2.11) we see that there is a locally Hölder continuous function which coincides with $u \mu$-almost everywhere. This implies that every point $x \in X$ is a Lebesgue point of $u$. This is consistent with the fact that even singletons have positive capacity when $p>Q$. Then we consider the case $1<p \leq Q$. The proof is rather straightforward adaptation of the Euclidean argument after having the capacitary weak type estimate and the estimate for the capacity of the set where the function is large, see for example the proof of Theorem 1 on pages 161-162 in [5].

Theorem 4.5 Suppose that $u \in M^{1, p}(X)$ with $1<p \leq Q$. Then there is $E \subset X$ such that $\mathrm{C}_{p}(E)=0$ and

$$
\lim _{r \rightarrow 0} \int_{B(x, r)} u d \mu=u^{*}(x)
$$

exists for every $x \in X \backslash E$. Moreover

$$
\lim _{r \rightarrow 0} f_{B(x, r)}\left|u-u^{*}(x)\right|^{\kappa p} d \mu=0
$$

for every $x \in X \backslash E$ with $1 \leq \kappa<Q /(Q-p)$ and the function $u^{*}$ is the p-quasicontinuous representative of $u$.

Proof. Since continuous functions are dense in $M^{1, p}(X)$, we may choose $u_{i} \in C(X) \cap M^{1, p}(X)$ such that

$$
\left\|u-u_{i}\right\|_{M^{1, p}(X)}^{p} \leq 2^{-i(p+1)} \quad \text { for } i=1,2, \ldots
$$

Denote $A_{i}=\left\{x \in X M\left(u-u_{i}\right)(x)>2^{-i}\right\}$ for $i=1,2, \ldots$ Lemma 4.4 implies that

$$
\mathrm{C}_{p}\left(A_{i}\right) \leq c 2^{i p}\left\|u-u_{i}\right\|_{M^{1, p}(X)}^{p} \leq c 2^{-i}
$$


Clearly

$$
\left|u_{i}(x)-u_{B(x, r)}\right| \leq f_{B(x, r)}\left|u_{i}(x)-u_{i}\right| d \mu+f_{B(x, r)}\left|u_{i}-u\right| d \mu,
$$

which implies that

$$
\limsup _{r \rightarrow 0}\left|u_{i}(x)-u_{B(x, r)}\right| \leq M\left(u_{i}-u\right)(x) \leq c 2^{-i}
$$

when $x \in X \backslash A_{i}$. Let $B_{k}=\bigcup_{i=k}^{\infty} A_{i}, k=1,2, \ldots$ Then by subadditivity of the capacity we have

$$
\mathrm{C}_{p}\left(B_{k}\right) \leq \sum_{i=k}^{\infty} \mathrm{C}_{p}\left(A_{i}\right) \leq c \sum_{i=k}^{\infty} 2^{-i}
$$

If $x \in X \backslash B_{k}$ and $i, j \geq k$, then

$$
\begin{aligned}
\mid u_{i}(x)- & u_{j}(x)\left|\leq \limsup _{r \rightarrow 0}\right| u_{i}(x)-u_{B(x, r)} \mid \\
& \quad+\limsup _{r \rightarrow 0}\left|u_{B(x, r)}-u_{j}(x)\right| \leq c\left(2^{-i}+2^{-j}\right) .
\end{aligned}
$$

Hence $\left\{u_{i}\right\}$ converges uniformly in $X \backslash B_{k}$ to a continuous function $v$. Now

$$
\limsup _{r \rightarrow 0}\left|v(x)-u_{B(x, r)}\right| \leq\left|v(x)-u_{i}(x)\right|+\limsup _{r \rightarrow 0}\left|u_{i}(x)-u_{B(x, r)}\right|
$$

so that

$$
v(x)=\lim _{r \rightarrow 0} f_{B(x, r)} u d \mu=u^{*}(x)
$$

for every $x \in X \backslash B_{k}$. Define $C=\bigcap_{k=1}^{\infty} B_{k}$. Then

$$
\mathrm{C}_{p}(C) \leq \lim _{k \rightarrow \infty} \mathrm{C}_{p}\left(B_{k}\right)=0
$$

and

$$
\lim _{r \rightarrow 0} f_{B(x, r)} u d \mu=u^{*}(x)
$$

exists for every $x \in X \backslash C$. This completes the proof of the first claim.

To prove the second claim, let $g_{u} \in D(u) \cap L^{p}(X)$ and

$$
D=\left\{x \in X \limsup _{r \rightarrow 0} r^{p} f_{B(x, r)} g_{u}^{p} d \mu>0\right\} .
$$

Lemma 4.3 shows that $\mathrm{C}_{p}(D)=0$. By the Sobolev-Poincaré inequality (see (2.10)) we have

$$
\lim _{r \rightarrow 0} f_{B(x, r)}\left|u-u_{B(x, r)}\right|^{\kappa p} d \mu=0
$$

for every $x \in X \backslash D$. 
We observe that

$$
\begin{aligned}
& \lim _{r \rightarrow 0}\left(f_{B(x, r)}\left|u-u^{*}(x)\right|^{\kappa p} d \mu\right)^{1 /(\kappa p)} \\
& \leq \lim _{r \rightarrow 0}\left(f_{B(x, r)}\left|u-u_{B(x, r)}\right|^{\kappa p} d \mu\right)^{1 /(\kappa p)}+\lim _{r \rightarrow 0}\left|u_{B(x, r)}-u^{*}(x)\right|=0
\end{aligned}
$$

whenever $x \in X \backslash(C \cup D)$ and $\mathrm{C}_{p}(C \cup D)=0$.

The final claim follows by fixing $\varepsilon>0$ and choosing $k$ large enough so that $\mathrm{C}_{p}\left(B_{k}\right)<\varepsilon / 2$. Then by outer regularity of the capacity there is an open set $O$ containing $B_{k}$ so that $\mathrm{C}_{p}(O)<\varepsilon$. Since $\left\{u_{i}\right\}$ converges uniformly to $u^{*}$ on $X \backslash O$ we conclude that $\left.u^{*}\right|_{X \backslash O}$ is continuous.

\section{References}

[1] Buckley, S.: Is the maximal function of a Lipschitz function continuous? Ann. Acad. Sci. Fenn. Math. 24 (1999), 519-528.

[2] Buörn, J., Macmanus, P. And Shanmugalingam, N.: Fat sets and pointwise boundary estimates for $p$-harmonic functions in metric spaces. J. Anal. Math. 85 (2001), 339-369.

[3] Cheeger, J.: Differentiability of Lipschitz functions on metric measure spaces. Geom. Funct. Anal. 9 (1999) 428-517.

[4] Coifman, R. R. And Weiss, G.: Analyse Harmonique Non-Commutative sur Certain Espaces Homogenés. Lecture Notes in Mathematics 242, Springer-Verlag, 1971.

[5] Evans, L.C. And Gariepy, R.F.: Measure Theory and Fine Properties of Functions. CRC Press, 1992.

[6] Fabes, E.B., Kenig, C.E. and Serapioni, R.P.: The local regularity of solutions of degenerate elliptic equations Comm. Partial Differential Equations 7 (1982), 77-116.

[7] Federer, H. and Ziemer, W. P.: The Lebesgue set of a function whose distribution derivatives are $p$-th power integrable. Indiana Univ. Math. J. 22 (1972), 139-158.

[8] HajŁasz, P.: Sobolev spaces on an arbitrary metric space. Potential Anal. 5 (1995), 403-415.

[9] Hajeasz, P. and Kinnunen, J.: Hölder quasicontinuity of Sobolev functions on metric spaces. Rev. Mat. Iberoamericana 14 (1998), 601-622.

[10] Hajzasz, P. and Koskela, P.: Sobolev meets Poincaré. C. R. Acad. Sci. Paris Sér. I Math. 320 (1995), 1211-1215.

[11] HajŁasz, P. And Koskela, P.: Sobolev met Poincaré. Mem. Amer. Math. Soc. 145 (2000), 1-101. 
[12] Heinonen, J. , Kilpeläinen, T. and Martio, O.: Nonliear Potential Theory of Degenerate Elliptic Equations. Oxford University Press, Oxford, 1993.

[13] Heinonen, J. And Koskela, P.: Quasiconformal maps in metric spaces with controlled geometry. Acta Math. 181 (1998), 1-61.

[14] Kilpeläinen, T.: On the uniqueness of quasi continuous functions. Ann. Acad. Sci. Fenn. Math. 23 (1998), 261-262.

[15] Kilpeläinen, T.: Weighted Sobolev spaces and capacity. Ann. Acad. Sci. Fenn. Math. 19 (1994), 95-113.

[16] Kinnunen, J.: The Hardy-Littlewood maximal function of a Sobolev function. Israel J. Math. 100 (1997), 117-124.

[17] Kinnunen, J. And Martio, O.: The Sobolev capacity on metric spaces. Ann. Acad. Sci. Fenn. Math. 21 (1996), 367-382.

[18] Macías, R.A. and Segovia, C.: A decomposition into atoms of distributions on spaces of homogeneous type. Adv. Math. 33 (1979), 271-309.

[19] Maz'ya, V.G. And Khavin, V.P.: Nonlinear potential theory. Russian Math. Surveys 27 (1972), 71-148.

[20] Semmes, S.: Finding curves on general spaces through quantitative topology with applications to Sobolev and Poincaré inequalities. Selecta Math. (N.S.) 2 (1996), 155-295.

[21] Shanmugalingam, N.: Newtonian spaces: An extension of sobolev spaces to metric measure spaces. Rev. Mat. Iberoamericana 16 (2000), 243-279.

[22] Ziemer, W. P.: Weakly differentiable functions. Springer-Verlag, 1989.

Recibido: 12 de febrero de 2001

Juha Kinnunen

Institute of Mathematics, P.O. Box 1100 FIN-02015 Helsinki University of Technology

Finland

juha.kinnunen@hut.fi

Visa Latvala

Department of Mathematics

University of Joensuu, P.O.Box 111

FIN-80101 Joensuu, Finland

visa.latvala@joensuu.fi 